\title{
Manipulación del precio del comercio internacional de productos del cobre: un análisis de los casos de Chile y el Perú ${ }^{1}$
}

\author{
Michael Hanni y Andrea Podestá
}

\section{Resumen}

Aquí se examina el grado de subfacturación de las exportaciones de productos del cobre - concentrados de cobre y cátodos de cobre refinado- de Chile y el Perú. Mediante la aplicación de una metodología novedosa en la que se toman en cuenta los términos contractuales típicos de la industria y los costos del seguro y el flete, se pone de manifiesto la existencia de transacciones comerciales de cobre a precios unitarios inferiores al valor de mercado. A partir de 2011 se observa una reducción importante del monto subfacturado, lo que refleja las mejoras en el ámbito de la tributación internacional en ambos países; no obstante, estos avances solo representan los primeros pasos hacia un marco legal y una institucionalidad suficientemente fuerte para fiscalizar la actividad minera de forma integral. Dado lo complejo que es evaluar y fiscalizar las transacciones mineras, se destaca la importancia de fortalecer las administraciones tributarias y aduaneras.

\section{Palabras clave}

Cobre, industria del cobre, exportaciones, precios, facturas, ilegalidad, tributación, administración fiscal, estudios de caso, Chile, Perú

\section{Clasificación JEL}

$$
\text { H26, H32 y Q37 }
$$

\section{Autores}

Michael Hanni es Oficial de Asuntos Económicos de la División de Desarrollo Económico en la Comisión Económica para América Latina y el Caribe (CEPAL). Correo electrónico: michael.hanni@cepal.org.

Andrea Podestá es Consultora de la División de Desarrollo Económico en la Comisión Económica para América Latina y el Caribe (CEPAL). Correo electrónico: apodestae@gmail.com.

\footnotetext{
En este documento se profundiza y amplía el informe del proyecto conjunto de la Comisión Económica para América Latina y el Caribe (CEPAL) y la Deutsche Gesellschaft für Internationale Zusammenarbeit (GIZ) titulado "Flujos financieros ilícitos, la evasión fiscal y el sector extractivo". Dicho proyecto formó parte del programa global de la cooperación alemana Lucha contra los Flujos Financieros llícitos, financiado por el Ministerio Federal de Cooperación Económica y Desarrollo (BMZ) de Alemania. Los autores agradecen los comentarios y sugerencias de un árbitro anónimo.
} 


\section{Introducción}

Las pérdidas tributarias asociadas con la explotación y la comercialización de materias primas es un tema clave para los países en desarrollo. La desaceleración de la economía mundial y la merma de los precios internacionales de estos productos - en particular del petróleo crudo y de los minerales y los metales - con respecto a los niveles alcanzados durante el auge han provocado una caída importante de los ingresos públicos. Esta situación ha llevado a muchos países a abordar asignaturas pendientes relacionadas con la fiscalización de las transacciones internacionales de materias primas, que podrían costarles millones de dólares en términos de ingresos no percibidos.

La investigación de este fenómeno es aún incipiente, pero ha adquirido un interés creciente para los gobiernos y el público en general porque los estudios que se han realizado confirman que la dimensión del problema podría ser grande. Por ejemplo, la Comisión Económica para África encontró que las pérdidas de capital debidas a los flujos financieros ilícitos que salen de ese continente han crecido significativamente en los últimos años y se concentran en transacciones comerciales de materias primas, sobre todo de petróleo crudo, minerales y metales (CEPA, 2015). En un informe de la Conferencia de las Naciones Unidas sobre Comercio y Desarrollo (UNCTAD, 2016) se sugiere que algunos países en desarrollo pierden hasta el $67 \%$ del valor de las materias primas que exportan debido a la facturación fraudulenta del comercio internacional.

La reducción de las pérdidas tributarias ocasionadas por la manipulación del precio del comercio internacional de las materias primas representa un gran desafío para los países en desarrollo. Como plantea la Organización de Cooperación y Desarrollo Económicos (OCDE, 2015), existen importantes brechas en términos de datos sobre la fijación de precios en el sector minero, lo que hace difícil que las administraciones tributarias detecten transacciones atípicas y comprueben que el precio de una transacción es fraudulento. A esto se debe sumar la falta de desarrollo de las capacidades en el ámbito de la tributación internacional que presentan las administraciones tributarias de muchos países en desarrollo (Readhead, 2016).

En este contexto, resulta interesante examinar algunos casos específicos de América Latina. En particular, en esta investigación se propone analizar el grado de subfacturación de las exportaciones de productos del cobre - concentrados de cobre y cátodos de cobre refinado- de Chile y el Perú. Ambos países desempeñan un papel clave en la producción y el comercio de estos productos a nivel mundial, aunque la composición de sus exportaciones presenta matices que podrían influir en el grado de subfacturación de cada país. Según la Comisión Chilena del Cobre (COCHILCO, 2016), el $44,8 \%$ del volumen de las exportaciones chilenas de cobre fino corresponden a productos refinados, lo que contrasta con una participación del 17,6\% en el caso peruano. En cambio, los concentrados de cobre representan el 47,3\% de las exportaciones en Chile y el 82,0\% en el Perú2. Al mismo tiempo, la actividad cuprífera representa una importante fuente de valor agregado, inversión, divisas e ingresos públicos en ambos países. Por lo tanto, cualquier manipulación del precio del comercio podría tener impactos económicos relevantes.

Con el fin de analizar una posible manipulación del precio del comercio internacional de estos productos mineros, se utiliza una fuente de datos muy rica: las declaraciones aduaneras de las empresas exportadoras registradas por las administraciones nacionales de aduanas de ambos países. En estas declaraciones se incluye información sobre las empresas involucradas, así como los valores y volúmenes exportados. Además, especialmente en el caso de Chile, se brindan datos sobre la composición del producto comercializado. Esto permite cruzar información de transacciones

\footnotetext{
2 El volumen restante de las exportaciones de cobre fino en ambos países corresponde a otros productos de fundición, que representan el 7,9\% del total en Chile y el 0,4\% en el Perú.
} 
efectuadas a un precio atípico por un determinado exportador, con información de los reportes anuales de ese exportador donde se detallan los términos contractuales que rigen la venta de la producción.

El grado de subfacturación de las exportaciones se estima a partir de una metodología basada en Hong, Pak y Pak (2014), en que se compara el precio unitario implícito de una materia prima exportada con el precio observado en el mercado internacional. Este estudio contribuye a la literatura gracias a la introducción de ciertas mejoras metodológicas novedosas. En primer lugar, se incorpora en el cálculo del filtro de precio una modelación de la fijación típica del precio de cada producto. En segundo lugar, en vez de aplicar una deducción del 10\% al precio de mercado para determinar el límite inferior de este, se calcula el precio de mercado en términos FOB (franco a bordo), aplicando a cada producto en ambos países los costos del flete y el seguro obtenidos de las declaraciones aduaneras de Chile.

Los resultados de este análisis confirman la existencia de transacciones donde el precio unitario implícito declarado por el exportador difiere sustancialmente del valor que cabe esperar tomando en cuenta el precio de mercado y los contratos referenciales de cada producto. Sin embargo, resulta importante destacar que, en el transcurso del período analizado, el monto subfacturado cayó tanto en términos absolutos como relativos. Este resultado puede deberse al fortalecimiento de los marcos regulatorios relacionados con la tributación internacional, ya que en ambos países se han refinado las reglas antievasión, particularmente en el ámbito de los precios de transferencia.

Este documento se ordena de la siguiente manera. En la sección II se examina la estructura de la actividad cuprífera, el modo en que este sector se organiza en Chile y el Perú, y cómo se posiciona en la industria mundial. A continuación, en la sección III se brinda una breve descripción de las principales metodologías que se emplean para estimar las manipulaciones del precio del comercio internacional. Luego, en la sección IV se presenta la metodología que se aplica en este estudio, así como los resultados obtenidos en relación con el comercio de productos del cobre de Chile y el Perú. Por último, en la sección $\vee$ se exponen algunas conclusiones y reflexiones finales.

\section{Producción y comercialización del cobre}

La producción primaria del cobre empieza con la extracción del mineral a través de la minería a cielo abierto, la minería subterránea o la lixiviación (véase el diagrama 1). El mineral de cobre no se suele exportar, dado que la mayor parte es ganga que no tiene valor comercial (OCDE, 2015). Dependiendo del tipo de mineral extraído - oxidado o sulfurado - se lleva a cabo uno de dos procesos de transformación. Los minerales oxidados generalmente pasan por el proceso hidrometalúrgico, donde se aplica lixiviación y electrodeposición para extraer el metal de cobre y producir cátodos de cobre refinado. Se estima que alrededor del $18 \%$ de la producción mundial de cobre refinado proviene de este proceso (ICSG, 2015). 


\section{Diagrama 1}

La cadena de transformación del cobre y sus productos clave

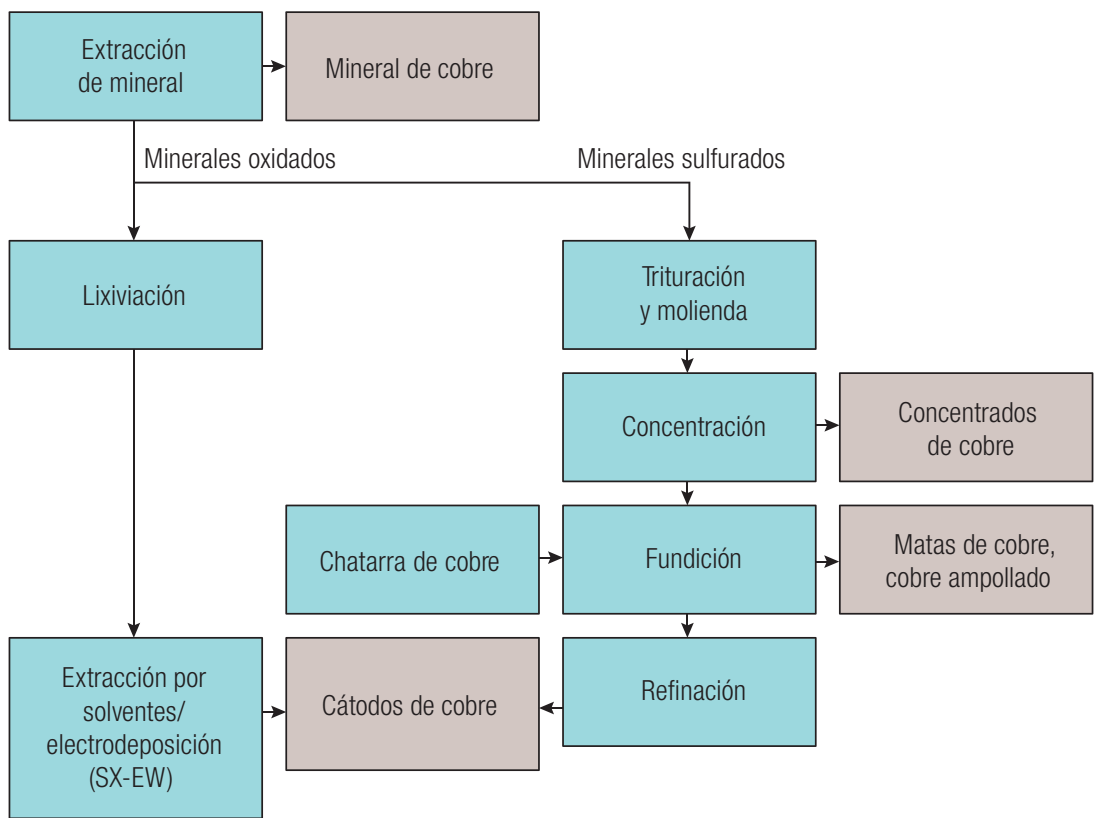

Fuente: Elaboración propia, sobre la base de Organización de Cooperación y Desarrollo Económicos (OCDE), "Addressing information gaps on prices of mineral products: mineral product pricing practices case studies: copper, gold and iron ore", París, 2015; y Grupo Internacional de Estudio sobre el Cobre (ICSG), The World Copper Factbook 2014, Lisboa, 2015.

A los minerales sulfurados, por su parte, se los somete a un proceso de trituración, molienda y flotación para obtener un concentrado que en general contiene entre un $20 \%$ y un $40 \%$ de metal de cobre, que se comercializa ampliamente en el mercado internacional. Luego, en la etapa de fundición, se obtienen matas de cobre (con una concentración del 50\% al 70\%) o cobre ampollado (con una concentración del 98,5\% al 99,5\%). Según la OCDE (2015), los productos que provienen de esta etapa se comercializan relativamente poco en el mercado internacional. Por último, el cobre fundido se refina mediante un proceso de fuego o de refinación electrolítica para obtener cátodos de cobre refinado con una pureza del 99,99\% o más. La producción de cobre refinado a través de estos procesos se exporta extensamente y representa alrededor del $64 \%$ de la producción mundial (ICSG, 2015).

Por otro lado, cabe destacar que la chatarra de cobre desempeña un papel importante en la producción de cobre refinado a nivel mundial. Se estima que la denominada producción secundaria, alimentada por el reciclaje de chatarra, representa alrededor del 18\% de la producción mundial de productos refinados de cobre (ICSG, 2015).

En este marco es posible analizar cómo se distribuye la producción de cobre en Chile y el Perú, así como la importancia relativa de ambos países en la producción mundial. Según estadísticas del Servicio Geológico de los Estados Unidos, Chile es el principal productor de cobre y, en 2015, concentraba alrededor del 30\% de la producción minera mundial. Como se ilustra en el cuadro 1, de los 5,76 millones de toneladas métricas (tm) de cobre fino producido en el país en dicho año, 3,99 millones correspondían a concentrados de cobre y 1,78 millones a cátodos refinados a través del proceso de extracción por solventes/electrodeposición. Una parte de la producción de concentrados se usó para producir cátodos de cobre (910.000 tm) por medio del proceso de refinación electrolítica, así como otros productos de fundición. De la producción total de cátodos del país, el 66\% se obtuvo mediante el proceso de extracción por solventes/electrodeposición y el 34\% mediante el de refinación electrolítica. 


\section{Cuadro 1}

Chile y Perú: indicadores de la producción y las exportaciones de cobre fino, 2015 (En miles de toneladas métricas y en porcentajes)

\begin{tabular}{|c|c|c|c|c|}
\hline \multirow[b]{2}{*}{ Producto } & \multicolumn{2}{|c|}{ Chile } & \multicolumn{2}{|c|}{ Perú } \\
\hline & $\begin{array}{l}\text { Miles de } \\
\text { toneladas } \\
\text { métricas }\end{array}$ & $\begin{array}{l}\text { Porcentaje } \\
\text { del total }\end{array}$ & $\begin{array}{l}\text { Miles de } \\
\text { toneladas } \\
\text { métricas }\end{array}$ & $\begin{array}{c}\text { Porcentaje } \\
\text { del total }\end{array}$ \\
\hline \multicolumn{5}{|c|}{ A. Producción } \\
\hline Producción minera de cobre fino & 5764 & $\ldots$ & 1705 & $\ldots$ \\
\hline Cátodos SX-EW (producto final) & 1778 & 30,9 & 73 & 4,3 \\
\hline Concentrados & 3986 & 69,1 & 1632 & 95,7 \\
\hline Productos de fundición (producto final) & 586 & 10,2 & 48 & 2,8 \\
\hline Cátodos E. R. (producto final) & 910 & 15,8 & 280 & 16,4 \\
\hline Concentrados (producto final) & 2489 & 43,2 & 1304 & 76,5 \\
\hline Total de cátodos SX-EW + E. R. & 2688 & 46,6 & 353 & 20,7 \\
\hline \multicolumn{5}{|c|}{ B. Exportaciones } \\
\hline Exportaciones de cobre fino & 5737 & $\ldots$ & 1628 & $\ldots$ \\
\hline Concentrados & 2714 & 47,3 & 1335 & 82,0 \\
\hline Productos de fundición & 455 & 7,9 & 7 & 0,4 \\
\hline Cátodos & 2568 & 44,8 & 286 & 17,6 \\
\hline
\end{tabular}

Fuente: Elaboración propia, sobre la base de Comisión Chilena del Cobre (COCHILCO), Anuario de Estadísticas del Cobre y Otros Minerales, 1996-2015, Santiago, 2016; y Ministerio de Energía y Minas, Anuario Minero, 2015, Lima, 2016.

Nota: SX-EW se refiere al proceso de extracción por solventes/electrodeposición; E. R. se refiere al proceso de refinación electrolítica.

En cuanto a las exportaciones chilenas, estas llegaron a 5,74 millones de tm de cobre fino, de las que 2,57 millones (44,8\% del total) correspondieron a cátodos refinados, 2,72 millones (47,3\%) a concentrados de cobre, y el resto $(7,9 \%)$ a otros productos de fundición ${ }^{3}$. Resulta interesante destacar la alta concentración de empresas privadas que participaron en las exportaciones de concentrados de cobre (87,9\% del total en 2015), lo que contrasta con la participación de la empresa estatal Codelco, que fue de solo el 12,1\%. Esto se refleja también en la composición de las exportaciones totales de las empresas privadas, en las que la participación de los concentrados de cobre fue del $56,8 \%$. En el caso de Codelco, en cambio, este producto representó el 21,4\% del total.

El Perú, por su parte, fue el tercer productor de cobre a nivel mundial en 2015, con una producción levemente inferior a la de China. Como se puede apreciar en el cuadro 1, la producción minera de cobre fino del país alcanzó 1,70 millones de toneladas métricas (alrededor del 9\% del total mundial). La mayor parte tomó la forma de concentrados $-1,63$ millones de tm-, dado que la producción de cátodos a través del proceso de extracción por solventes/electrodeposición solo alcanzó 73.000 tm (pero representó el 20\% de la producción total de cátodos). Una parte de la producción de concentrados de cobre se usó para producir cátodos por medio del proceso de refinación electrolítica, y la producción alcanzó 280.000 tm (80\% del total). Resulta importante subrayar que la capacidad para fundir y refinar cobre es limitada en el país, lo que se refleja en el alto porcentaje de la producción total que se destina a los concentrados (el $76,5 \%$ en el Perú frente al 43,2\% en Chile). Durante el mismo año se exportaron 1,63 millones de tm de cobre fino: 1,34 millones de tm de concentrados (82,0\%) y 286.000 tm de cátodos (17,6\%).

\footnotetext{
3 Vale remarcar que el volumen de producción y el volumen de exportación en un año determinado no necesariamente coinciden, debido a rezagos en el proceso de producción que pueden dar como resultado un desfasaje de esta con respecto a las exportaciones.
} 
En ambos países se destaca el hecho de que la producción minera está muy concentrada en un grupo limitado de empresas. En Chile, los cinco productores principales concentraron el $75 \%$ de la producción total en 2015 (COCHILCO, 2016). De igual manera, la producción minera de las cinco empresas principales del Perú representó el $81 \%$ de la producción total (Ministerio de Energía y Minas, 2016). De particular importancia para este estudio es el hecho de que la mayoría de estas firmas son filiales o empresas conjuntas de multinacionales. En Chile, tres de los cinco productores principales son controlados por empresas multinacionales, en tanto que, en el Perú, esta situación se da en los cinco productores principales ${ }^{4}$.

En el cuadro 2 se ilustra el importante papel que desempeñan Chile y el Perú en el comercio mundial de productos del cobre. En 2015, estos países representaban conjuntamente el 50\% del valor total de las exportaciones de mineral de cobre y sus concentrados. En términos del comercio internacional de cátodos de cobre refinado, Chile era con creces el principal exportador mundial, con una cuota de mercado del 34\%. El Perú, en cambio, se ubicó en noveno lugar con una participación del $4 \%$, lo que refleja principalmente su limitada capacidad para fundir y refinar.

\section{Cuadro 2}

Valor de las exportaciones mundiales de productos seleccionados de cobre y participación de los principales países exportadores, 2015

(En millones de dólares y en porcentajes)

A. Mineral de cobre y sus concentrados (HS6 260300)

\begin{tabular}{lcc}
\hline País & Valor & Participación \\
\hline Chile & 13891 & 34 \\
\hline Perú & 6568 & 16 \\
\hline Australia & 3659 & 9 \\
\hline Estados Unidos & 3083 & 8 \\
\hline Canadá & 3001 & 7 \\
\hline Mongolia & 2280 & 6 \\
\hline Brasil & 1984 & 5 \\
\hline México & 1159 & 3 \\
\hline España & 1131 & 3 \\
\hline Filipinas & 700 & 2 \\
\hline Otros países & 3237 & 8 \\
\hline Total & 40693 & 100
\end{tabular}

B. Cátodos de cobre refinado (HS6 740311)

Fuente: Elaboración propia, sobre la base de cifras de la Base de Datos Estadísticos de las Naciones Unidas sobre el Comercio de Productos Básicos (COMTRADE).

\footnotetext{
4 En Chile, los productores controlados por empresas multinacionales son los siguientes: Minera Escondida (BHP Billiton es el propietario mayoritario); Compañía Minera Doña Inés de Collahuasi (propiedad conjunta de Anglo American plc, Glencore y Japan Collahuasi Resources B.V.), y Anglo American Sur (Anglo American plc es el propietario mayoritario). En el Perú, dichos productores son los siguientes: Minera Antamina (propiedad conjunta de BHP Billiton, Glencore, Teck y Mitsubishi Corporation); Southern Peru Copper Corporation (Grupo México S.A.B. de C.V.); Minera Cerro Verde (Freeport-McMoRan es el propietario mayoritario); Minera Antapaccay (Glencore), y Minera Chinalco Perú (Aluminum Corporation of China).
} 
Este liderazgo en el comercio internacional del cobre también conlleva un impacto importante en las economías de ambos países. En Chile, las exportaciones de productos del cobre representaron en promedio el 47\% del valor total de las exportaciones entre 2011 y 2015. En el Perú, la participación del cobre fue menor, con un promedio del $23 \%$ en el mismo período, lo que refleja la diferencia entre el nivel de producción de estos países y la oferta más diversificada del Perú en términos de productos mineros. Entre 1995 y 2015, las exportaciones de productos del cobre generaron 418.000 millones de dólares en Chile y 96.000 millones en el Perú5.

\section{Breve descripción de las metodologías que permiten detectar la manipulación de los precios comerciales}

Históricamente, el análisis sobre la manipulación del precio del comercio internacional se ha basado en el enfoque del país socio impulsado por Bhagwati (1964 y 1974), que parte de la premisa de que las importaciones del país $A$ que provienen del país $B$ son también las exportaciones del país $B$ que van hacia el país $A$. Si luego de ajustar por los gastos del seguro y el flete (entre otras variables), el valor de las importaciones registradas en un determinado país es superior al valor que el resto del mundo ha registrado como exportaciones hacia dicho país, se dice que existe una sobrefacturación de las importaciones. Del mismo modo, si el valor ajustado de las exportaciones contabilizadas en un país es inferior a lo que otros países han declarado como importaciones desde ese país, significa que existe una subfacturación de las exportaciones.

Una serie de estudios recientes basados en este enfoque ha alimentado una nueva corriente en la literatura, que tiene por objeto cuantificar el grado de facturación fraudulenta del comercio internacional (la sobrefacturación de las importaciones y la subfacturación de las exportaciones), como componente clave de los flujos financieros ilícitos que salen de los países en desarrollo. En particular, se destacan los informes de Global Financial Integrity (Kar y Spanjers, 2015; Hollingshead, 2010), de la Comisión Económica para África (CEPA, 2015) y de la Comisión Económica para América Latina y el Caribe (CEPAL, 2016) sobre los flujos financieros ilícitos asociados con la manipulación del precio del comercio internacional.

Un supuesto implícito en este método es que los valores declarados en el país socio son los valores de plena competencia. Ello ofrece la ventaja de que no es necesario determinar el precio de plena competencia para cada transacción (como en el método del filtro de precios), pero puede distorsionar el grado de facturación fraudulenta cuando el precio declarado en el país socio tampoco es el de libre mercado. Las estimaciones basadas en este enfoque permiten detectar las salidas de capital únicamente cuando uno de los socios declara un precio, cantidad o calidad diferente de los que registra el otro en relación con los bienes comercializados. Sin embargo, podrían existir ocasiones en que tanto el exportador como el importador registren el mismo precio relativo a un bien, pero que este se encuentre lejos del precio de mercado.

Otro factor a causa del cual los cálculos efectuados con este método pueden estar subestimados es la utilización de datos agregados por país, socio o producto. Esto se debe a que cada par de registros de país socio incluye múltiples operaciones que pueden compensarse entre sí debido a que algunas pueden estar sobrevaluadas y otras subvaluadas. Otro inconveniente son las asimetrías en las estadísticas bilaterales del comercio internacional, que pueden originarse en distintas fuentes, entre ellas: las diferencias en la valoración de las importaciones y las exportaciones (CIF y FOB); las

5 Según cifras de la Base de Datos Estadísticos de las Naciones Unidas sobre el Comercio de Productos Básicos (COMTRADE). 
diferencias en la clasificación o la nomenclatura de los productos, y las diferencias en la atribución de los socios comerciales y en los registros de reexportación (Javorsek, 2016).

En la metodología del filtro de precio impulsada por Zdanowicz, Pak y Sullivan (1999) y Pak, Zanakis y Zdanowicz (2003) se busca detectar las transacciones del comercio internacional que se alejan de un rango de precios considerados normales, de mercado o de libre competencia, y estimar así su desviación. Este rango de precios normales puede determinarse mediante dos enfoques principales: el análisis del rango intercuartil o el uso del precio de libre mercado.

En el primer enfoque se utilizan los registros de importación y exportación declarados ante las autoridades aduaneras con el fin de identificar los precios de comercio anormales. Se calcula el precio implícito del cuartil superior y el del cuartil inferior en relación con cada categoría de productos, y se considera de plena competencia el rango de precios situados entre los dos anteriores. La manipulación de los precios se evalúa al contrastar cada registro de la base de datos comerciales con el rango intercuartil asociado a la clasificación correspondiente de un producto. Si el precio declarado se ubica dentro de dicho rango, se supone que se trata de una transacción en condiciones de plena competencia, es decir, de una transacción normal. Si el precio está por encima del precio del cuartil superior, se estaría ante una sobrefacturación del comercio, y si se sitúa por debajo del valor del cuartil inferior, se estaría ante una subfacturación.

Sin embargo, en la literatura se destacan ciertas falencias y limitaciones de este método. Una de ellas tiene relación con su endogeneidad, ya que el precio de los cuartiles se estima a partir de todas las transacciones comerciales, entre las que siempre existirán operaciones con precios por debajo del cuartil inferior y por encima del superior, aun cuando todas las transacciones se realicen a precios de plena competencia. Los filtros de precios intercuartiles también se ven afectados por las transacciones que se llevan a cabo entre empresas vinculadas y se registran en la base de datos comerciales, por lo que si este tipo de transacciones son muy frecuentes, el rango de precios estimado puede no ser representativo del rango de libre competencia.

Otro problema tiene lugar cuando se trata de productos muy heterogéneos, debido a que este enfoque puede llevar a clasificar erróneamente transacciones legítimas de productos de alta calidad como sobrevaloradas, transacciones de libre competencia de productos de baja calidad como subvaluadas, y transacciones a precios anormales de productos de calidad media como legítimas. En el caso de los productos con precios muy volátiles, si el rango intercuartil de precios se estima sobre la base de registros anuales, no se tendrán en cuenta las variaciones mensuales de los precios y ello dará como resultado estimaciones menos precisas.

Al igual que en el método del país socio, otra dificultad surge cuando se usan registros agrupados en una misma categoría de productos, en vez de datos de cada despacho aduanero, ya que en esa agrupación se pueden incluir transacciones tanto sobrevaluadas como subvaluadas que se compensan entre sí, lo que llevaría a subestimar el grado de manipulación de los precios del comercio. También se pueden subestimar los montos de la facturación fraudulenta cuando se trata de transacciones que involucran grandes cantidades de un bien en las que se registra un precio marginalmente diferente al de libre competencia. En esas situaciones, como el precio de la operación se ubica dentro del rango intercuartil, esta es clasificada como normal, a pesar de que el valor total de la facturación errónea podría ser muy significativo.

Por otro lado, en el enfoque del filtro de precio basado en el precio de libre mercado se calculan los montos sobre- o subfacturados comparando los precios unitarios implícitos en los registros aduaneros con un precio (o rango de precios) que se considera de libre competencia y que es publicado por diversos mercados y organismos. Cuando el precio de la importación o la exportación se desvía del precio de libre mercado, se considera que esa transacción refleja una falsificación del precio del comercio. 
Para la mayoría de los bienes es sumamente difícil determinar un verdadero precio de mercado, el llamado precio arm's-length o de plena competencia. Sin embargo, en el caso de algunas materias primas existen mercados suficientemente líquidos y con la transparencia necesaria para cotizar el precio de libre mercado 6 .

Según Hong, Pak y Pak (2014), el enfoque del filtro de precio de libre mercado tiene ciertas ventajas, como el hecho de que no es necesario estimar el precio de plena competencia, ya que se utiliza el precio de libre mercado publicado por ciertas instituciones. Además, a diferencia del filtro de precio intercuartil, el de libre mercado tiene en cuenta la variación mensual de los precios del producto y es independiente de los valores de importación declarados, por lo que el registro de las operaciones entre partes relacionadas no sesga las estimaciones. Sin embargo, los precios de libre mercado no están disponibles en relación con todos los productos; incluso en los casos en que existen mercados de productos básicos con precios fácilmente identificables puede ocurrir que estos no sean representativos del valor final que obtiene el exportador debido a los términos de referencia incluidos en los contratos de cada industria. Esto era lo que ocurría en el caso de las estimaciones realizadas en el presente estudio, donde, además del precio internacional del bien, se consideraron los contratos referenciales.

\section{Estimación del grado de manipulación del precio de comercio de los productos del cobre en Chile y el Perú}

\section{Metodología}

En este estudio se aplica la metodología del filtro de precios utilizada por Hong, Pak y Pak (2014) y Grondona y Burgos (2015) para estimar el valor subfacturado de las exportaciones de productos del cobre. A diferencia de lo que ocurre en la mayoría de los estudios existentes basados en un filtro intercuartil de precios, esos autores comparan el precio unitario de las transacciones de un bien específico - plátanos, en el primer estudio, y productos de la soja, en el segundo- con el precio referencial de ese bien en el mercado mundial. Este método se parece a los que se usan para calcular los precios de transferencia en las transacciones entre partes relacionadas, como el denominado sexto método o una variante del método del precio comparable no controlado. La presente investigación contribuye a la literatura porque, a diferencia de los estudios mencionados, en ella se consideran dos ajustes relevantes que otorgan mayor precisión a las estimaciones. En primer lugar, se incluye un modelo de fijación de precios de acuerdo con las condiciones contractuales que se aplican en la industria a los productos analizados. Además, para calcular el precio mensual de mercado en términos FOB se consideran los costos del flete y el seguro que se incluyen en las declaraciones aduaneras, en vez de asumir una deducción constante del 10\%.

Las principales fuentes de datos son las declaraciones aduaneras de exportación de productos del cobre en ambos países y los precios de mercado del cobre, el oro y la plata. La información sobre las ventas de productos mineros al exterior proviene de la base de datos de Penta-Transaction, que contiene las declaraciones aduaneras de los exportadores de estos bienes que están registradas en las agencias de aduanas. En general, en estas declaraciones se incluye el nombre del exportador, la fecha de embarcación, el valor FOB, y el peso bruto y neto del bien exportado. En el caso de Chile, 6 Véase, por ejemplo, Hong, Pak y Pak (2014), donde se calcula el grado de facturación fraudulenta del comercio de plátanos
contrastando el precio de libre mercado de ese bien con los precios del registro de las importaciones estadounidenses. 
contienen asimismo datos sobre la composición del producto y los costos del seguro y el flete, es decir, se registra también el valor CIF.

Los precios de mercado provienen de la base de datos de la UNCTAD, en la que se presentan los valores mensuales de los principales productos básicos exportados. Generalmente, estos precios se expresan en términos CIF, representan el valor acordado en los principales mercados (como la Bolsa de Metales de Londres) y están asociados a un cierto estado del producto. Por ejemplo, en el caso del cobre, el precio se refiere a los cátodos de cobre refinado con una ley mínima de cobre del 99,99\% (cobre de grado A).

En el caso de las exportaciones de mineral de cobre y sus concentrados, el filtro de precio se construye sobre la base del precio de mercado y los términos contractuales típicos que rigen la venta de este producto en el mercado internacional. Como se explica en Teck (2015) y en Nyrstar (2016), los contratos relativos a la venta de concentrados de cobre suelen regirse por los términos referenciales anuales establecidos entre los principales mineros y fundidores. El precio recibido por parte de la empresa minera se fija según las ecuaciones que se indican a continuación.

En primer lugar, se calcula el precio del metal que hay en el concentrado:

$$
\text { Precio del metal }=\left((M \star D) \star P_{m}\right)+\left(\left(O_{m}{ }^{\star} D_{O m}\right) \star P_{O m}\right),
$$

donde $M$ se refiere a la ley del metal en el concentrado (habitualmente entre el $20 \%$ y el $30 \%$ en el caso de los concentrados de cobre); $D$ se refiere al porcentaje del valor del metal pagado después de aplicar una deducción estándar para cubrir las pérdidas de metal en el proceso de fundición (las mineras típicamente reciben entre el $96,5 \%$ y el $96,75 \%$ del valor del metal), y $P_{m}$ es el precio de mercado del cobre. En los contratos también se suelen contemplar los pagos asociados al contenido de oro o plata si la concentración de estos supera cierto valor mínimo. En este caso, $O_{m}$ se refiere al contenido del otro metal en el concentrado, sea oro o plata; $D_{O m}$ se refiere al porcentaje de ese metal que se paga (alrededor del $90 \%$ ), y $P_{O m}$ es el precio de mercado del metal.

En segundo lugar, se calculan las deducciones por el procesamiento del concentrado:

$$
\text { Deducciones }=T C R C+P P+R C_{O m}+\text { Otros }
$$

En este caso, TCRC se refiere al cargo básico de tratamiento y al cargo de refinación (TC y $\mathrm{RC}$, respectivamente), que en teoría son los costos de transformar el concentrado en metal refinado. PP corresponde a la participación del fundidor en el movimiento del precio de mercado, es decir a las escalas de ajustes crecientes o decrecientes (escalators y de-escalators). Esta participación se calcula mensualmente como un valor fijo por tonelada que se suma al cargo de tratamiento por cada dólar, si el precio de mercado está por encima del precio básico. En cambio, cuando el precio de mercado está por debajo del precio básico, del cargo de tratamiento se deduce un monto fijo por tonelada. Sin embargo, desde 2005-2006 las principales empresas mineras no aceptan este componente en los contratos referenciales. $R C_{O m}$ se refiere a los cargos de refinación del contenido del otro metal -sea plata u oro - en el concentrado. En el ítem Otros se contemplan otros cargos, que incluyen multas por niveles elevados de contaminantes en el concentrado, pero, dada la falta de información precisa sobre el nivel de contaminantes, este componente no puede incluirse en el análisis.

Finalmente, el precio neto del mercado que se expresa en términos CIF se calcula como el precio del metal que hay en el concentrado menos las deducciones correspondientes acordadas en el contrato:

$$
\text { Precio neto del mercado }=\text { precio del metal }- \text { deducciones }
$$


Entonces, el monto subfacturado en el comercio de los concentrados de cobre se estima a partir de la siguiente ecuación:

$$
\text { Monto subfacturado }=\text { volumen }{ }^{\star} M A X\left(0, P_{*}-P\right)
$$

En este caso, $P$ es el precio unitario implícito derivado de la declaración aduanera, usando el peso neto que excluye el contenido de humedad en el concentrado ${ }^{7}$. $P_{*}$ es igual al Precio neto del mercado (obtenido en la ecuación (3)), expresado en términos de toneladas métricas secas menos los costos del flete y el seguro. Estos costos se calcularon mensualmente a partir de las declaraciones chilenas que incluyen información sobre el valor CIF de las exportaciones. Al Perú se le aplicaron los mismos valores, asumiendo que estos son similares en los dos países.

Lamentablemente, no en todas las declaraciones se incluyen datos precisos sobre la composición del producto exportado. Esto ocurre sobre todo en el caso del Perú y complica los cálculos porque las empresas mineras reciben un pago basado en el contenido de metal que hay en el producto. Por eso, respecto de ambos países se presentan tres filtros de precios en los que se suponen diferentes leyes, para cubrir los valores que suelen usarse en la industria. En el caso de Chile, debido a que las declaraciones contienen más datos, se presenta también una estimación en la que se toma en cuenta la composición registrada.

En el caso de las exportaciones de cátodos de cobre refinado, el monto subfacturado se calcula usando una ecuación similar a la que proponen Hong, Pak y Pak (2014), dado que se supone que la composición del producto exportado se aproxima a la del producto en el que se basa el precio de referencia internacional. A diferencia de lo que hicieron dichos autores, aquí se estima el precio mensual de mercado, en términos FOB, aplicando los costos del seguro y el flete obtenidos de las declaraciones aduaneras de Chile. Estos mismos valores se usaron para el Perú.

\section{Resultados relativos al mineral de cobre y sus concentrados}

Como se aprecia en el gráfico 1, existe una correlación entre los tres filtros de precio y la evolución de los precios unitarios implícitos en las declaraciones aduaneras, lo que sugiere que la mayoría de las transacciones de venta de concentrados de cobre obedece a los términos referenciales de la industria. Durante el período comprendido entre 2006 y el primer semestre de 2016, las 7.872 declaraciones registradas en Chile correspondieron a 72,10 millones de toneladas métricas netas, con un valor FOB de 140.273 millones de dólares. En el Perú, las 9.142 declaraciones registradas correspondieron a 35,11 millones de toneladas métricas netas con un valor FOB de 61,9 millones de dólares. El precio unitario implícito de estas declaraciones era de 1,95 dólares por kilogramo en Chile y de 1,76 dólares por kilogramo en el Perú.

\footnotetext{
7 En ambos países, cuando el peso neto era igual al peso bruto, se aplicó un ajuste del 10\% al peso registrado para obtener una estimación del peso en términos de toneladas métricas secas.
} 


\section{Gráfico 1}

Chile y Perú: precio unitario implícito en las declaraciones de exportación de minerales de cobre y sus concentrados (HS6 260300), y filtros de precios basados en tres escenarios, $2006-2016^{a}$

(En dólares por tonelada métrica neta)

\section{A. Chile}

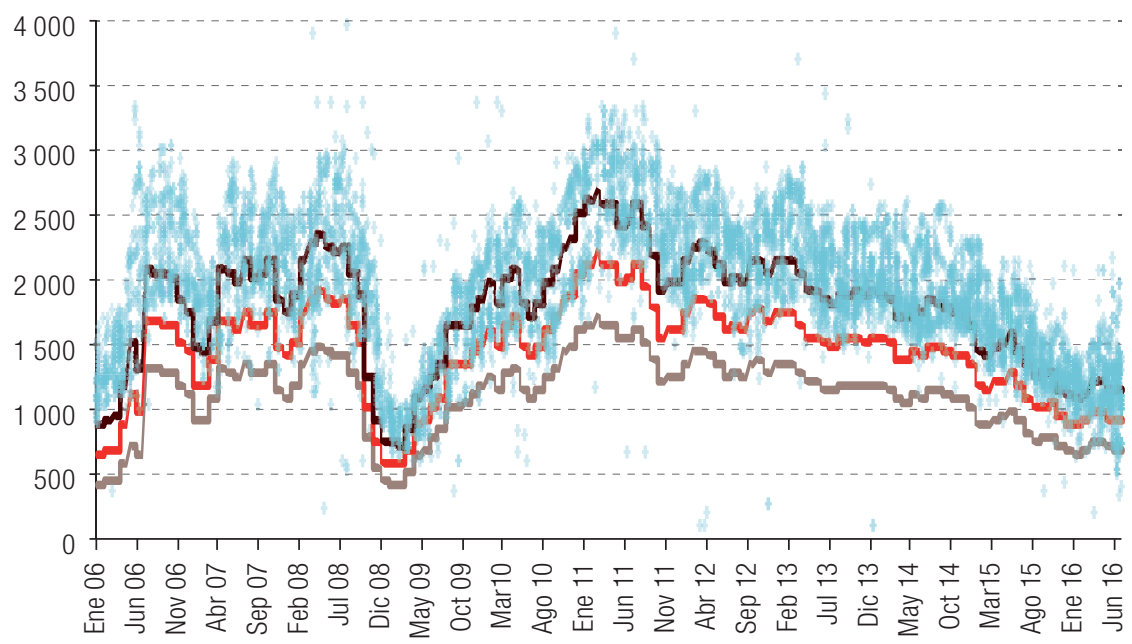

\section{B. Perú}

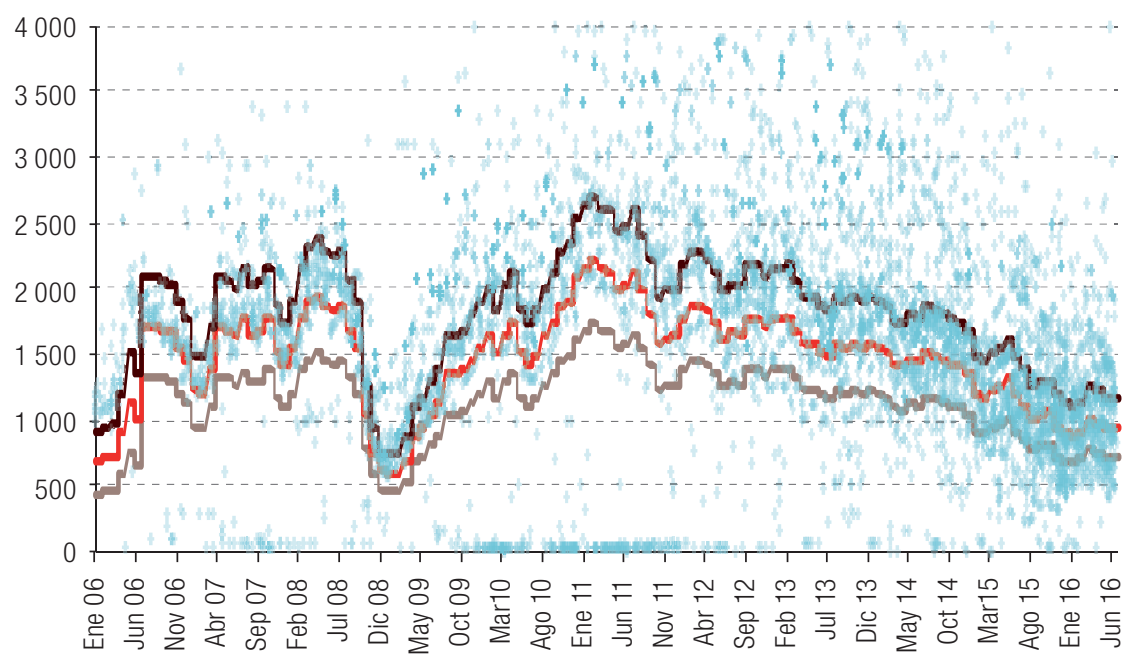

+ Precios observados - Filtro de precio (ley de 20\%)

— Filtro de precio (ley de 25\%) — Filtro de precio (ley de 30\%)

Fuente: Elaboración propia, sobre la base de cifras de Penta-Transaction.

a Al primer semestre de 2016.

Existen transacciones que se sitúan por encima -incluso a veces muy por encima- de las líneas que marcan los filtros de precio. En estos casos es muy probable que el concentrado exportado incluya una cantidad de plata u oro que aumente el precio unitario de la transacción. Como se mencionó anteriormente, en los contratos típicos relativos a la venta de los concentrados de cobre se contemplan pagos por estos metales si su concentración sobrepasa cierto nivel. Aunque en las declaraciones aduaneras del Perú no se suele detallar la composición del bien exportado, en la 
mayoría de las chilenas sí, lo que permite confirmar la existencia de un contenido importante de oro (alrededor de 2 gramos por tonelada métrica en promedio) y de plata (alrededor de 98 gramos por tonelada métrica en promedio).

Sin embargo, se detectan diferencias importantes entre las observaciones de ambos países. En el caso de Chile, los precios observados tienden a mantenerse dentro de un rango más o menos estable en el transcurso del tiempo. En efecto, la mayoría de los datos se sitúan por encima de las líneas de los filtros de precio asociados con cualquiera de las leyes de cobre consideradas, lo que refleja una cierta homogeneidad del producto en términos del contenido de cobre y de metales preciosos. En cambio, los precios unitarios provenientes de las declaraciones aduaneras peruanas tienen una gran dispersión, lo que sugiere que la composición del producto exportado es muy heterogénea. Además, en el caso del Perú existe una cantidad importante de transacciones que se ubican por debajo de todos los filtros de precio.

En el Perú se destaca un número de transacciones en las que se registran precios unitarios cercanos a 0 dólares por tonelada. Una revisión de estas declaraciones sugiere que la gran mayoría corresponden a exportaciones por camión en la frontera con Chile. Aunque en las declaraciones no se especifica la composición del producto, es muy probable que correspondan a mineral de cobre y no a concentrado. Por lo tanto, estas operaciones tendrían un valor esperado mucho menor que el filtro adoptado y no necesariamente representarían manipulaciones del precio del comercio. Cabe mencionar que los volúmenes de estas transacciones son pequeños en términos relativos, sobre todo los de las exportaciones por barco hacia China, por lo que su participación en las estimaciones de los montos subfacturados es cercana a cero.

El otro grupo de transacciones peruanas que llaman la atención corresponde al período 2015-2016. En particular, destacan las declaraciones de exportación de la Minera Chinalco Perú S. A., que empezó a exportar concentrados de cobre de su nueva mina Toromocho en 2014. En dichas declaraciones se observa un valor unitario inferior al filtro de precio asociado a una ley del $20 \%$. Aunque en la mayoría de esas declaraciones aduaneras no se especifica la concentración de cobre, a partir de las pocas que sí contienen información se puede suponer que en general se exporta un concentrado que tiene una ley de entre el $20 \%$ y el $22 \%$, lo que coincide con el escenario de la ley del $20 \%$. Sin embargo, resulta importante destacar que en la producción de esta nueva mina hay un alto nivel de contaminación (CHINALCO-CMC, 2016). Sus concentrados contienen mayores niveles de arsénico, lo que podría tener un impacto negativo en el precio obtenido en el mercado. Es posible que la empresa haya vendido su producción a precios más bajos y que, por lo tanto, estas transacciones no necesariamente correspondan a manipulaciones del precio. Sin embargo, debido a la falta de información disponible, no ha sido factible realizar ese ajuste en las estimaciones.

En el cuadro 3 se muestran las estimaciones del monto subfacturado correspondientes a las exportaciones de concentrados de cobre de ambos países. En Chile, durante el período analizado, el valor de las exportaciones subvaluadas alcanzó 3.035 millones de dólares, que equivalen al 2,2\% del valor FOB exportado, de acuerdo con la estimación en la que se considera la composición del concentrado que consta en las declaraciones (línea "registrado"). La mayor parte de este monto corresponde al subperíodo 2006-2010, cuando el valor subfacturado representó en promedio el 4,0\% del valor exportado. En los años siguientes se observa una reducción del monto subfacturado, tanto en términos absolutos como en términos relativos, con un promedio del 0,9\% del valor exportado entre 2011 y 2015. 


\section{Cuadro 3}

Chile y Perú: valor FOB y monto subfacturado de las exportaciones de concentrados de cobre (HS6 260300), basado en tres escenarios, 2006-2016ª

(En millones de dólares y en porcentajes)

A. Chile

\begin{tabular}{|c|c|c|c|c|c|c|c|c|c|c|c|c|}
\hline & 2006 & 2007 & 2008 & 2009 & 2010 & 2011 & 2012 & 2013 & 2014 & 2015 & 2016 & Total \\
\hline \multicolumn{13}{|c|}{ En millones de dólares } \\
\hline Ley del 20\% & 0 & 0 & 0 & 0 & 0 & 0 & 0 & 0 & 0 & 0 & 0 & 0 \\
\hline Ley del 25\% & 7 & 53 & 62 & 106 & 104 & 33 & 49 & 6 & 4 & 17 & 61 & 504 \\
\hline Ley del 30\% & 157 & 326 & 496 & 522 & 746 & 271 & 560 & 305 & 285 & 229 & 331 & 4229 \\
\hline Registrado & 389 & 553 & 549 & 383 & 401 & 111 & 254 & 85 & 137 & 74 & 98 & 3035 \\
\hline Valor (FOB) & 11376 & 13476 & 12866 & 7629 & 12375 & 14462 & 15912 & 17190 & 16475 & 12949 & 5564 & 140273 \\
\hline \multicolumn{13}{|c|}{ En porcentajes del valor exportado (FOB) } \\
\hline Ley del 20\% & 0,0 & 0,0 & 0,0 & 0,0 & 0,0 & 0,0 & 0,0 & 0,0 & 0,0 & 0,0 & 0,0 & 0,0 \\
\hline Ley del 25\% & 0,1 & 0,4 & 0,5 & 1,4 & 0,8 & 0,2 & 0,3 & 0,0 & 0,0 & 0,1 & 1,1 & 0,4 \\
\hline Ley del 30\% & 1,4 & 2,4 & 3,9 & 6,8 & 6,0 & 1,9 & 3,5 & 1,8 & 1,7 & 1,8 & 5,9 & 3,0 \\
\hline Registrado & 3,4 & 4,1 & 4,3 & 5,0 & 3,2 & 0,8 & 1,6 & 0,5 & 0,8 & 0,6 & 1,8 & 2,2 \\
\hline \multicolumn{13}{|c|}{ B. Perú } \\
\hline & 2006 & 2007 & 2008 & 2009 & 2010 & 2011 & 2012 & 2013 & 2014 & 2015 & 2016 & Total \\
\hline \multicolumn{13}{|c|}{ En millones de dólares } \\
\hline Ley del 20\% & 1 & 4 & 11 & 1 & 6 & 7 & 5 & 12 & 11 & 35 & 24 & 118 \\
\hline Ley del 25\% & 25 & 38 & 63 & 36 & 100 & 98 & 123 & 99 & 113 & 263 & 124 & 1083 \\
\hline Ley del 30\% & 278 & 518 & 523 & 311 & 590 & 582 & 683 & 536 & 601 & 848 & 467 & 5936 \\
\hline Valor (FOB) & 2794 & 4348 & 4666 & 3842 & 6248 & 7814 & 8044 & 7112 & 6934 & 6570 & 3535 & 61906 \\
\hline \multicolumn{13}{|c|}{ En porcentajes del valor exportado (FOB) } \\
\hline Ley del 20\% & 0,0 & 0,1 & 0,2 & 0,0 & 0,1 & 0,1 & 0,1 & 0,2 & 0,2 & 0,5 & 0,7 & 0,2 \\
\hline Ley del 25\% & 0,9 & 0,9 & 1,4 & 0,9 & 1,6 & 1,3 & 1,5 & 1,4 & 1,6 & 4,0 & 3,5 & 1,8 \\
\hline Ley del 30\% & 9,9 & 11,9 & 11,2 & 8,1 & 9,4 & 7,5 & 8,5 & 7,5 & 8,7 & 12,9 & 13,2 & 9,6 \\
\hline
\end{tabular}

Fuente: Elaboración propia, sobre la base de cifras de Penta-Transaction.

a Al primer semestre de 2016.

En el Perú, si se toma el escenario intermedio en que se asume una ley del 25\%, se observa que el valor subfacturado llegó a 1.083 millones de dólares durante el período 2006-2016, lo que equivale al 1,8\% del valor FOB exportado. El monto subfacturado muestra una tendencia creciente, con un alza muy importante en 2015, tanto en términos absolutos como relativos, debido en parte a las exportaciones de la nueva mina mencionadas anteriormente (cuyo valor subfacturado fue de 96,3 millones de dólares en 2015). Además, alrededor del 50\% del monto subfacturado acumulado durante el período analizado (538 millones de dólares) corresponde a ventas donde es posible que la empresa esté vendiendo un concentrado con un contenido de cobre inferior al $25 \%$, dado que se informa que sus operaciones en el continente corresponden a concentraciones de cobre de entre el $20 \%$ y el 30\%. Lamentablemente, los datos disponibles en las declaraciones aduaneras no permiten confirmar esta suposición. Sin embargo, si se considera el escenario en que la ley es del $20 \%$, el monto subfacturado acumulado de esta empresa durante el período tiende a cero.

Como era de esperar, los montos subfacturados se concentran en el comercio con los principales compradores de concentrados de cobre de ambos países (véase el cuadro 4). En Chile, las exportaciones hacia los cinco países socios más importantes representan casi el 85\% del valor total y, a su vez, en estos países se concentra el 78,2\% del monto subfacturado estimado. En general, los montos subfacturados relacionados con estos socios tuvieron un peso relativo al valor exportado bastante similar, que va del $1,9 \%$ al $2,1 \%$, salvo en el caso de la República de Corea $(1,4 \%)$ y de la India (2,5\%). 


\section{Cuadro 4}

Chile y Perú: monto subfacturado de las exportaciones de concentrados de cobre (HS6 260300), por socio, 2006-2016

(En millones de dólares y en porcentajes)

A. Chile (valores registrados)

\begin{tabular}{lccc}
\hline País & $\begin{array}{c}\text { Valor } \\
\text { subfacturado }\end{array}$ & Valor F0B & $\begin{array}{c}\text { Valor } \\
\text { subfacturado } \\
\text { relativo al } \\
\text { valor F0B }\end{array}$ \\
\hline Japón & 821 & 41713 & 2,0 \\
\hline China & 767 & 39764 & 1,9 \\
\hline India & 456 & 18087 & 2,5 \\
\hline República de Corea & 165 & 11613 & 1,4 \\
\hline Brasil & 163 & 7940 & 2,1 \\
\hline Otros países & 661 & 21156 & 3,1 \\
\hline Total & 3035 & 140273 & 2,2 \\
\hline
\end{tabular}

B. Perú (escenario con una ley del 25\%)

\begin{tabular}{lccc}
\hline País & $\begin{array}{c}\text { Valor } \\
\text { subfacturado }\end{array}$ & Valor FOB & $\begin{array}{c}\text { Valor } \\
\text { subfacturado } \\
\text { relativo al } \\
\text { valor FOB }\end{array}$ \\
\hline China & 358 & 25020 & 1,4 \\
\hline Japón & 332 & 10329 & 3,2 \\
\hline Alemania & 20 & 5991 & 0,3 \\
\hline República de Corea & 82 & 3291 & 2,5 \\
\hline Chile & 45 & 2726 & 1,6 \\
\hline Otros países & 247 & 14549 & 1,7 \\
\hline Total & 1083 & 61906 & 1,8 \\
\hline
\end{tabular}

Fuente: Elaboración propia, sobre la base de cifras de Penta-Transaction.

a Al primer semestre de 2016.

En el Perú, según el escenario en que se supone una ley del 25\%, los cinco países socios principales concentraron el $77,2 \%$ del monto subfacturado y, al mismo tiempo, el $76,5 \%$ del valor total exportado. Sin embargo, se observan importantes diferencias entre estos países en cuanto al peso relativo del monto subfacturado: los valores más elevados corresponden al Japón $(3,2 \%)$ y a la República de Corea (2,5\%). En cambio, en el comercio con Chile y con China, el principal socio, se registraron valores intermedios del 1,6\% y el 1,4\% del valor FOB, respectivamente ${ }^{8}$. Por último, se observa un peso relativo muy bajo en el comercio con Alemania $(0,3 \%)$.

\section{Resultados relativos a los cátodos refinados de cobre}

En el gráfico 2 se puede apreciar que la mayoría de los precios observados, obtenidos de las declaraciones sobre la exportación de cátodos de cobre refinado en Chile y el Perú, están en línea con el filtro de precio. Entre 2006 y el primer semestre de 2016 se registraron 83.767 declaraciones de exportación de cobre refinado en Chile y 12.172 en el Perú. En el primer país, el valor de las exportaciones ascendió a 199.585 millones de dólares, que correspondió a un volumen de 29,29 millones de toneladas métricas. Por otro lado, las declaraciones registradas en el Perú tuvieron un valor de 22.713 millones de dólares correspondientes a un volumen de 3,32 millones de toneladas métricas. En promedio, el precio unitario implícito se ubicó en 6,8 dólares por kilogramo en ambos países.

\footnotetext{
8 Dado que la capacidad de fundición y refinación de cobre es limitada en el Perú, parte de la producción de mineral y concentrados de cobre se exporta a Chile y se incorpora al proceso de refinación de ese país.
} 


\section{Gráfico 2}

Chile y Perú: precio unitario implícito en las declaraciones de exportación de cobre refinado (HS6 740311) y filtro de precio, 2006-2016a

(En dólares por kilogramo neto)

\section{A. Chile}

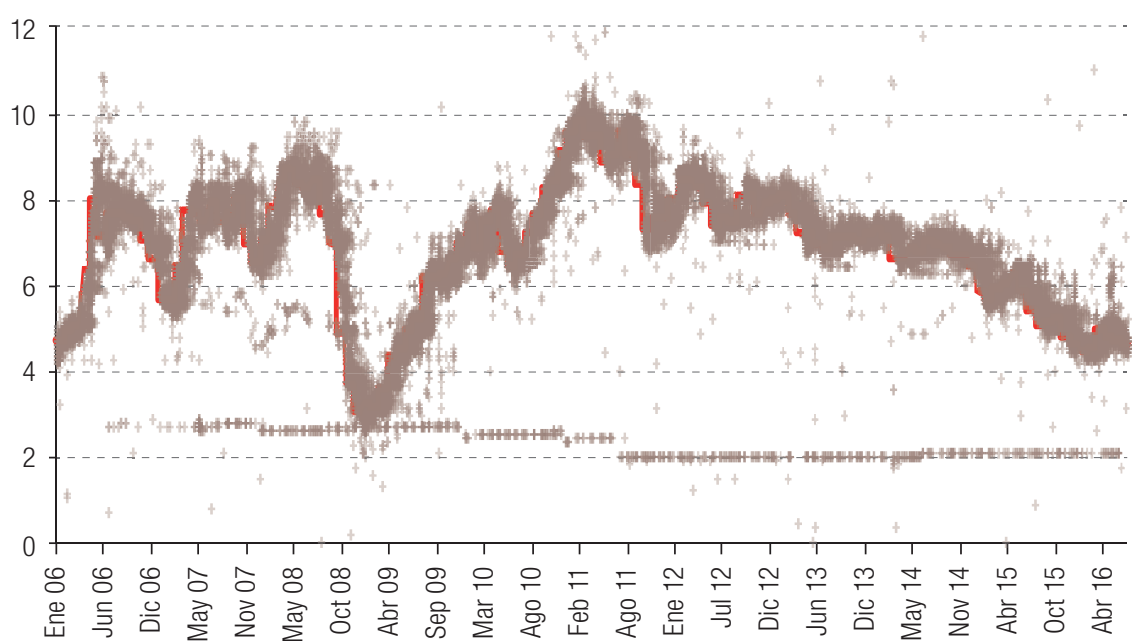

\section{B. Perú}

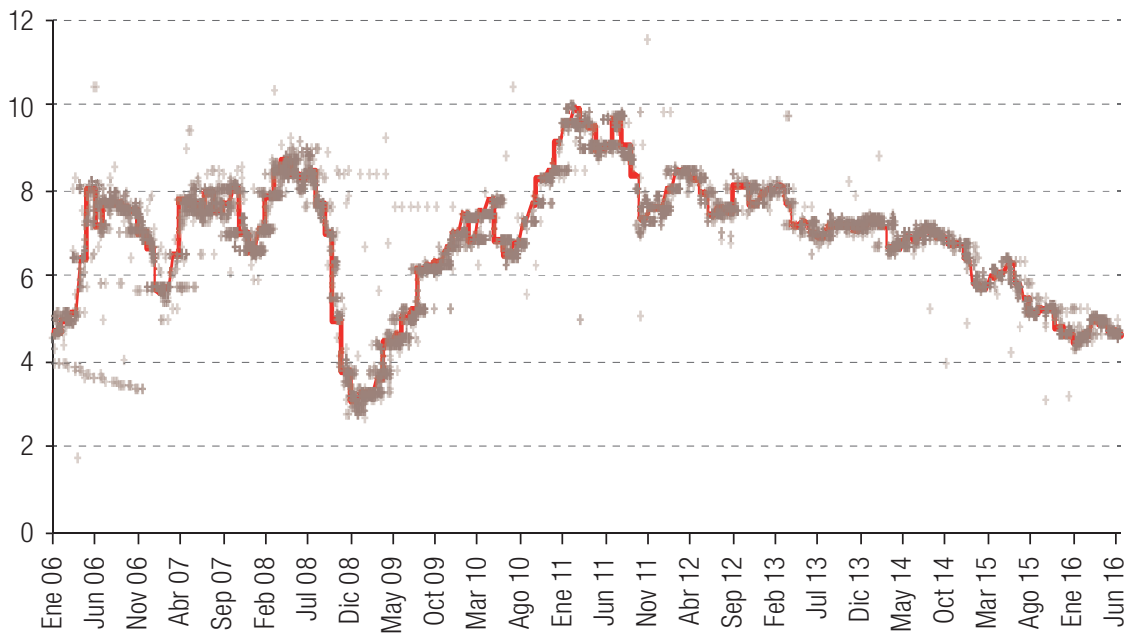

+ Precios observados $\quad$ Filtro de precio

Fuente: Elaboración propia, sobre la base de cifras de Penta-Transaction.

a Al primer semestre de 2016.

Sin embargo, se aprecia que en ambos países existen ciertas transacciones atípicas que no obedecen a la tendencia del precio de mercado. En Chile se nota una larga serie de transacciones que trazan una línea casi recta situada bastante por debajo del precio de mercado durante todo el período. Una revisión de ellas revela que corresponden a ventas de Codelco a clientes de China. Esto se debe a que, en mayo de 2005, Codelco firmó un contrato con China Minmetals Corporation (Minmetals) por la venta de 836.250 toneladas de cátodos durante un período de 15 años (180 meses). En el contrato se estipulaba que el precio de venta se determinaría mediante una parte fija reajustable y una parte variable que dependería del precio de mercado en el momento del 
embarque. Las exportaciones realizadas en virtud de este contrato empezaron en junio de 2006 y se canalizaron a través de la empresa Copper Partners Investment Company Ltd (Bermudas); esa compañía y Codelco participaban en partes iguales. Cabe mencionar que, en 2016, Codelco terminó su participación en esa empresa conjunta y puso fin al contrato con Minmetals, luego de que el Servicio de Impuestos Internos de Chile (SII) cuestionara el régimen tributario de la empresa conjunta y obligara a Codelco a pagar 149 millones de dólares en 2015 (CODELCO, 2016).

En el Perú, especialmente al comienzo del período, se nota la existencia de transacciones cuyo precio observado se mantiene por debajo del valor de mercado durante varios meses (véase el gráfico 2). Entre enero y noviembre de 2006, los precios observados de un grupo de operaciones en particular trazan una línea recta decreciente. Estas transacciones corresponden a ventas de cátodos de una empresa filial de una firma estadounidense a clientes de ese país. Lamentablemente, en las declaraciones no se incluye información sobre el comprador, por lo que no ha sido posible confirmar si estas ventas fueron entre partes relacionadas o no. Lo que llama la atención es que sus precios unitarios mostraron una tendencia a la baja, mientras el precio de mercado aumentaba sustancialmente (el $61 \%$ entre enero y mayo de 2006).

En contraste, otros grupos de transacciones atípicas que presentan una tendencia lineal suelen mostrar un precio fijo, es decir, trazan una línea horizontal. Por ejemplo, en 2007, en ciertas exportaciones de Southern Peru Copper Corporation se registraron precios similares durante ese año, a pesar de que el precio de mercado estuvo bastante inestable. En este último caso, en el informe de auditoría de esta empresa correspondiente a dicho año se señala que se solía vender cobre en virtud de contratos anuales y, por lo tanto, esto podría explicar la aparición de transacciones a un mismo precio durante un año (Southern Peru Copper Corporation, 2008).

En el cuadro 5 se muestran los montos subfacturados asociados con la exportación de cátodos de cobre refinado en Chile y el Perú. En el caso del primero, se estima que este valor alcanzó 6.348 millones de dólares entre 2006 y el primer semestre de 2016, lo que equivale al 3,2\% del valor exportado. De ese monto, 2.516 millones de dólares (el 40\% del total) corresponden a transacciones realizadas en virtud del contrato de largo plazo entre Codelco y Minmetals. El monto subfacturado relacionado con otras transacciones fue de 3.833 millones de dólares durante el período (equivalente al 1,9\% del valor exportado). En este último caso se observa una clara tendencia a la baja durante el período: el monto subfacturado pasó de un promedio de 567 millones de dólares por año entre 2006 y 2010, a un promedio de 182 millones de dólares anuales entre 2011 y 2015. Como resultado, el peso del monto subfacturado en relación con el valor exportado cayó de un promedio del 3,1\% en el primer subperíodo al 0,9\% en el segundo. En el Perú, el monto subfacturado alcanzó 369 millones de dólares entre 2006 y el primer semestre de 2016, es decir, el 1,6\% del valor exportado. Cabe mencionar que, al igual que ocurrió en Chile, tanto el valor de las salidas como su peso relativo en el valor exportado experimentaron un descenso notable durante el período analizado.

Como en el caso del comercio de los concentrados de cobre, los montos subfacturados se focalizan en los principales compradores de cobre refinado de ambos países. En el cuadro 6 se ilustra que, en Chile, los cinco países socios principales representan el 72,0\% del valor exportado y el $82,2 \%$ del valor subfacturado. Se observa que el comercio con China generó la mayor parte del monto subfacturado $(59,4 \%)$, impulsado principalmente por el efecto del contrato entre Codelco y Minmetals. Como resultado, el peso del monto subfacturado en el comercio con China alcanzó el $5,3 \%$ del valor exportado durante el período. En cambio, el peso de las exportaciones no asociadas con este contrato se ubicó en el 1,8\% del valor FOB, lo que coincide con los valores que se observan en relación con otros países socios (en un rango de entre el 1,6\% y el 2,4\%). 


\section{Cuadro 5}

Chile y Perú: monto subfacturado del precio del comercio internacional de cobre refinado (HS6 740311), 2006-2016a

(En millones de dólares y en porcentajes)

A. Chile

\begin{tabular}{|c|c|c|c|c|c|c|c|c|c|c|c|c|}
\hline & 2006 & 2007 & 2008 & 2009 & 2010 & 2011 & 2012 & 2013 & 2014 & 2015 & 2016 & Total \\
\hline \multicolumn{13}{|c|}{ En millones de dólares } \\
\hline Cobre refinado & 564 & 862 & 767 & 771 & 935 & 645 & 628 & 424 & 334 & 291 & 127 & 6348 \\
\hline Codelco/Minmetals & 75 & 319 & 253 & 130 & 285 & 347 & 329 & 300 & 253 & 186 & 39 & 2516 \\
\hline Otros & 489 & 543 & 514 & 642 & 651 & 298 & 299 & 124 & 81 & 105 & 88 & 3833 \\
\hline Valor (FOB) & 16114 & 19544 & 20307 & 15054 & 22646 & 25727 & 22066 & 19655 & 17087 & 14651 & 6738 & 199590 \\
\hline \multicolumn{13}{|c|}{ En porcentajes del valor exportado (FOB) } \\
\hline Cobre refinado & 3,5 & 4,4 & 3,8 & 5,1 & 4,1 & 2,5 & 2,8 & 2,2 & 2,0 & 2,0 & 1,9 & 3,2 \\
\hline Codelco/Minmetals & 0,5 & 1,6 & 1,2 & 0,9 & 1,3 & 1,3 & 1,5 & 1,5 & 1,5 & 1,3 & 0,6 & 1,3 \\
\hline Otros & 3,0 & 2,8 & 2,5 & 4,3 & 2,9 & 1,2 & 1,4 & 0,6 & 0,5 & 0,7 & 1,3 & 1,9 \\
\hline
\end{tabular}

B. Perú

\begin{tabular}{lrrrrrrrrrrrrr}
\hline & 2006 & 2007 & 2008 & 2009 & 2010 & 2011 & 2012 & 2013 & 2014 & 2015 & 2016 & Total \\
\hline & & \multicolumn{8}{c}{ En millones de dólares } \\
\hline Cobre refinado & 73 & 48 & 49 & 54 & 71 & 33 & 22 & 6 & 3 & 7 & 2 & 369 \\
\hline Valor (FOB) & 3005 & 2372 & 2571 & 1817 & 2495 & 2790 & 1846 & 1834 & 1838 & 1511 & 635 & 22713 \\
\hline \multicolumn{10}{c}{ En porcentajes del valor exportado (FOB) } \\
\hline Cobre refinado & 2,4 & 2,0 & 1,9 & 3,0 & 2,8 & 1,2 & 1,2 & 0,3 & 0,2 & 0,5 & 0,2 & 1,6 \\
\hline
\end{tabular}

Fuente: Elaboración propia, sobre la base de cifras de Penta-Transaction.

a Al primer semestre de 2016.

\section{Cuadro 6}

Chile y Perú: monto subfacturado del comercio internacional de cobre refinado (HS6 740311), por socio, 2006-2016a

(En millones de dólares y en porcentajes)

\section{A. Chile}

\begin{tabular}{lccc}
\hline País & $\begin{array}{c}\text { Valor } \\
\text { subfacturado }\end{array}$ & Valor F0B & $\begin{array}{c}\text { Valor } \\
\text { subfacturado } \\
\text { relativo al } \\
\text { valor FOB }\end{array}$ \\
\hline China & 3768 & 71616 & 5,3 \\
\hline $\begin{array}{l}\text { sin Codelco/ } \\
\text { Minmetals }\end{array}$ & 1252 & 70350 & 1,8 \\
\hline Estados Unidos & 418 & 26174 & 1,6 \\
\hline Italia & 415 & 16993 & 2,4 \\
\hline República de Corea & 295 & 14807 & 2,0 \\
\hline $\begin{array}{l}\text { Taimán (provincia } \\
\text { china) }\end{array}$ & 324 & 14065 & 2,3 \\
\hline Otros países & 1128 & 55934 & 2,0 \\
\hline Total & 6348 & 199590 & 3,2 \\
\hline
\end{tabular}

B. Perú

\begin{tabular}{lccc}
\hline País & $\begin{array}{c}\text { Valor } \\
\text { subfacturado }\end{array}$ & Valor FOB & $\begin{array}{c}\text { Valor } \\
\text { subfacturado } \\
\text { relativo al } \\
\text { valor FOB }\end{array}$ \\
\hline China & 46 & 5251 & 0,9 \\
\hline Italia & 77 & 4873 & 1,6 \\
\hline Estados Unidos & 108 & 4608 & 2,3 \\
\hline Brasil & 61 & 3306 & 1,8 \\
\hline $\begin{array}{l}\text { Taiwán (provincia } \\
\text { china) }\end{array}$ & 34 & 2015 & 1,7 \\
\hline Otros países & 43 & 2659 & 1,6 \\
\hline Total & 369 & 22713 & 1,6 \\
\hline
\end{tabular}

Fuente: Elaboración propia, sobre la base de cifras de Penta-Transaction.

a Al primer semestre de 2016.

En el caso del Perú, los cinco países socios principales concentraron el 88,3\% del valor exportado y del monto subfacturado. En términos absolutos, el comercio con los Estados Unidos generó el mayor monto subfacturado durante el período (108 millones de dólares). En relación con 
este país también se observó que el monto subfacturado tenía un peso elevado en el valor FOB de las exportaciones $(2,3 \%)$, en comparación con otros países socios, cuyos valores se situaron entre el $0,9 \%$ y el $1,8 \%$. Cabe mencionar que la mayor parte del monto subfacturado asociado con los Estados Unidos correspondió a los primeros años del período (el 54\% del total en 2006 y 2007), lo que coincide con lo que se mencionó anteriormente al analizar el gráfico 2.

\section{Conclusiones}

Los resultados obtenidos en este estudio, a través de la aplicación de una metodología novedosa y adaptada a las condiciones locales de los países analizados, ponen de manifiesto la existencia de transacciones comerciales de cobre concentrado y refinado en las que se registran precios unitarios inferiores al valor de mercado (en el que se toman en cuenta los términos contractuales típicos de la industria, y los costos del flete y el seguro). Estas operaciones ameritan una fiscalización por parte de las autoridades correspondientes con el fin de confirmar si representan o no desvíos fraudulentos del precio. De esta forma, las autoridades podrían focalizar sus esfuerzos en diseñar e implementar medidas tendientes a reducir las pérdidas tributarias causadas por este tipo de operaciones. Además, dado que tanto en el caso de Chile como del Perú la actividad cuprífera es muy importante, el hecho de que la sociedad perciba una pérdida tributaria asociada con ella podría tener graves repercusiones en términos de la confianza en el sistema tributario de estos países.

Entre los principales resultados, se estima que el monto potencialmente subfacturado en el comercio de concentrados de cobre durante el período comprendido entre 2006 y el primer semestre de 2016 alcanzó 3.035 millones de dólares en Chile (el 2,2\% del valor exportado) y 1.083 millones de dólares en el Perú (el 1,8\% del valor exportado, en el escenario en que se supone una ley del $25 \%)$. Al mismo tiempo, los montos potencialmente subfacturados en el caso de las ventas de cátodos de cobre refinado llegaron a 3.833 millones de dólares en Chile (el 3,2\% del valor exportado) y 369 millones de dólares en el Perú (el 1,6\% del valor exportado) ${ }^{9}$.

Resulta importante mencionar que, en el transcurso del período analizado, el monto subfacturado cayó tanto en términos absolutos como relativos. En el caso de las exportaciones chilenas de concentrados de cobre, el monto subfacturado bajó de un promedio del 4,0\% del valor exportado en 2006-2010, a un promedio del 0,9\% entre 2011 y 2015. Del mismo modo, los montos subfacturados de las exportaciones de cobre refinado en ambos países se redujeron desde un promedio del 3,1\% en Chile y $2,4 \%$ en el Perú hasta un promedio del 0,9\% y el 0,7\%, respectivamente. Este resultado puede deberse al perfeccionamiento de los marcos regulatorios relacionados con la tributación internacional, debido a que en ambos países se han refinado las reglas contra la evasión y la elusión en los últimos años, particularmente en el ámbito de los precios de transferencia. No obstante, los avances logrados solo representan los primeros pasos hacia un marco legal y una institucionalidad suficientemente fuerte para fiscalizar esta actividad de manera integral en sus diferentes etapas, desde la mina hasta la comercialización en el mercado internacional.

Por otra parte, es de destacar las dificultades que se encontraron al estimar los montos subfacturados de las exportaciones de estos productos. Aunque se utilizaron las declaraciones

\footnotetext{
9 Sin embargo, estas estimaciones son más bien conservadoras, dado que en los registros de exportación pueden existir fuentes de subestimación que no se han podido ajustar debido a la falta de datos disponibles. Por ejemplo, en una investigación del Centro de Investigación Periodística (CIPER) de Chile se detectó que, por décadas, las exportaciones de concentrado de cobre de ese país no se habían controlado, y se había aceptado la pureza y el peso declarado por las mineras. Del mismo modo, Castillo (2015) asegura que la fiscalización de las exportaciones mineras es la gran debilidad del Servicio Nacional de Aduanas de ese país. De hecho, una comisión investigadora de la Cámara de Diputados de Chile concluyó que el país no cuenta con la institucionalidad pública adecuada para ejercer un control eficiente y riguroso que determine la trazabilidad del cobre, y llamó la atención acerca de la falta de atribuciones y recursos de los organismos fiscalizadores (Cámara de Diputados de Chile, 2015).
} 
aduaneras de las empresas exportadoras, que permiten un análisis mucho más detallado, en ellas no se suele incluir toda la información necesaria para calcular con precisión todos los pagos y deducciones relacionados con cada transacción. Además, a pesar de que se tuvo acceso a los informes financieros de varias empresas involucradas con transacciones subvaluadas, en ellos por lo general no se incluye información completa sobre los términos de los contratos que esas empresas suscriben con sus compradores. A este respecto, el análisis efectuado reafirma los desafíos que las administraciones tributarias y aduaneras enfrentan a la hora de fiscalizar las declaraciones juradas que presentan las empresas mineras en sus respectivos países. En este sentido, resulta importante avanzar hacia registros aduaneros que contengan información más detallada y completa, tanto en lo que respecta a la concentración, la composición y la características de cada producto, como a los datos del comprador (específicamente para determinar si se trata de transacciones entre empresas vinculadas), de manera tal que las autoridades puedan fiscalizar de forma más eficiente.

La labor futura en este ámbito debería centrarse en investigar otros canales a través de los cuales las empresas desvían de manera ilegal hacia el extranjero las utilidades que obtienen en el sector minero. Aunque la evidencia sugiere que una creciente proporción del comercio internacional del cobre se realiza en condiciones de mercado, en los países considerados todavía se podrían estar perdiendo ingresos tributarios por otros medios, sobre todo mediante transacciones entre las filiales - que desempeñan un papel importante en el sector minero de ambos países - y sus casas matrices en el extranjero. Si bien las operaciones entre partes relacionadas se rigen por las reglas de los precios de transferencia, en ellas muchas veces se suelen incluir pagos por servicios respecto de los cuales es muy difícil fijar un precio de mercado. Cabe resaltar que otra área de trabajo importante consiste en examinar la estructuración financiera de las empresas mineras con el objeto de determinar si esa estructura refleja un intento de reducir artificialmente los impuestos que se pagan. En particular, se debe profundizar la investigación del posible abuso de derivados financieros para trasladar beneficios fuera de la región (PWYP Norway, 2011).

Por último, en el combate de este tipo de prácticas resulta muy relevante el paquete amplio de medidas acordadas por los gobiernos en el marco del Proyecto OCDE/G20 sobre la Erosión de la Base Imponible y el Traslado de Beneficios (BEPS), como por ejemplo los informes país por país. Estos brindarán a las administraciones tributarias una visión global de las operaciones de las empresas multinacionales, ya que en ellos se deberá indicar dónde estas empresas declaran los beneficios, los impuestos y las actividades económicas. Esta información permitirá a las administraciones tributarias evaluar los riesgos en materia de precios de transferencia, así como de otras prácticas BEPS, a fin de focalizar los recursos de fiscalización en las áreas más críticas.

\section{Bibliografía}

Bhagwati, J. (1974), "Fiscal policies, the faking foreign trade declarations, and the balance of payments", Illegal Transactions in International Trade, J. N. Bhagwati (ed.), Amsterdam, North Holland.

(1964), "On the underinvoicing of imports", Bulletin of the Oxford University Institute of Economics and Statistics, vol. 26, No 4, Oxford, University of Oxford.

Cámara de Diputados de Chile (2015), "Informe de la Comisión Especial Investigadora encargada de analizar las eventuales responsabilidades de CODELCO y de ENAMI, con ocasión de la sustracción de concentrados de cobre", 3 de marzo [en línea] https://www.camara.cl/pdf.aspx?prmID=14119\&prmTI $\mathrm{PO}=$ INFORMECOMISION.

Castillo, R. (2015), El cobre: anatomía del mayor fraude minero en Chile, Santiago, Andros Impresores.

CEPA (Comisión Económica para África) (2015), Illicit Financial Flow. Report of the High Level Panel on Illicit Financial Flows from Africa, Addis Abeba.

CEPAL (Comisión Económica para América Latina y el Caribe) (2016), Estudio Económico de América Latina y el Caribe, 2016 (LC/G.2684-P), Santiago. 
CHINALCO-CMC (CHINALCO Mining Corporation International) (2016), Annual Report, 2015 [en línea] http:// www.hkexnews.hk/listedco/listconews/sehk/2016/0428/LTN20160428325.pdf.

COCHILCO (Comisión Chilena del Cobre) (2016), Anuario de Estadísticas del Cobre y Otros Minerales, 1996-2015, Santiago.

CODELCO (Corporación Nacional del Cobre de Chile) (2016), Memoria Anual, 2015, Santiago.

Grondona, V. y M. Burgos (2015), "Estimación de los precios de transferencia: el caso del complejo sojero", Documento de Trabajo, № 71, Buenos Aires, Centro de Economía y Finanzas para el Desarrollo de la Argentina (CEFID-AR).

Hollingshead, A. (2010), The Implied Tax Revenue Loss from Trade Mispricing, Washington, D.C., Global Financial Integrity.

Hong, K., C. Pak y S. Pak (2014), "Measuring abnormal pricing - an alternative approach: the case of US banana trade with Latin American and Caribbean countries", Journal of Money Laundering Control, vol. 17, № 2, Bingley, Emerald.

ICSG (Grupo Internacional de Estudio sobre el Cobre) (2015), The World Copper Factbook 2014, Lisboa.

Javorsek, M. (2016), "Asymmetries in international merchandise trade statistics: a case study of selected countries in Asia-Pacific", Working Paper Series, № SD/WP/02, Bangkok, Comisión Económica y Social para Asia y el Pacífico (CESPAP) [en línea] http://www.unescap.org/sites/default/files/SD_Working_Paper_ April2016_Asymmetries_in_International_Trade_Statistics.pdf.

Kar, D. y J. Spanjers (2015), Illicit Financial Flows from Developing Countries: 2004-2013, Washington, D.C., Global Financial Integrity.

Ministerio de Energía y Minas (2016), Anuario Minero, 2015, Lima.

Nyrstar (2016), "Modeling Nyrstar", agosto [en línea] http://www.nyrstar.com/investors/en/Investors\%20 Materials/English/Modeling\%20Nyrstar.pdf.

OCDE (Organización de Cooperación y Desarrollo Económicos) (2015), "Addressing information gaps on prices of mineral products: mineral product pricing practices case studies: copper, gold and iron ore", París.

Pak, S. J., S. H. Zanakis y S. J. Zdanowicz (2003), "Detecting abnormal pricing in international trade: the Greece-USA case", Interfaces, vol. 33, № 2, Catonsville, INFORMS.

PWYP Norway (Publish What You Pay Norway) (2011), "Protection from derivative abuse", diciembre [en línea] https://www.publishwhatyoupay.no/sites/all/files/1006a-PWYP_DerivativesReport_ENG_DOWNLOAD_0.pdf.

Readhead, A. (2016), Preventing Tax Base Erosion in Africa: a Regional Study of Transfer Pricing Challenges in the Mining Sector, Instituto para la Gobernanza de los Recursos Naturales.

Southern Peru Copper Corporation (2008), "Estados financieros, 31 de diciembre de 2007 y 31 de diciembre de 2006", Lima.

Teck (2015), "Modelling Workshop", noviembre [en línea] http://www.teck.com/media/Investors-PresentationsWebcasts-20151104-modelling-workshop.pdf.

UNCTAD (Conferencia de las Naciones Unidas sobre Comercio y Desarrollo) (2016), Trade Misinvoicing in Primary Commodities in Developing Countries: The Cases of Chile, Cote d'Ivoire, Nigeria, South Africa and Zambia (UNCTAD/SUC/2016/2), Ginebra.

Zdanowicz, J., S. Pak y M. Sullivan (1999), "Brazil-United States trade: capital flight through abnormal pricing", The International Trade Journal, vol. 13, № 4, Abingdon, Taylor \& Francis. 